\title{
Impact of predator on the host-vector disease model with stage structure for the vector
}

\author{
Fengyan Zhou ${ }^{1,2,3}$, Chengrong Ma ${ }^{4}$, Hongzhen Liang ${ }^{3,5}$, Binxiang Dai ${ }^{2}$ and Hongxing Yao ${ }^{*^{*}}$
}

\author{
"Correspondence: \\ hxyao@ujs.edu.cn \\ ${ }^{5}$ School of Finance and Economics, \\ Jiangsu University, Zhenjiang, \\ P.R. China \\ Full list of author information is \\ available at the end of the article
}

\begin{abstract}
In this paper, we propose a host-vector-predator model with stage structure for the vector to explore the impact of biological control agents on host-vector dynamics and disease control. Here the total vector population is divided into two physiological subclasses which are immature and mature subclasses. Holling type II functional response is used to portray the interactions between vectors and predators. Stability analysis of the equilibria demonstrates that the basic reproduction number gives the threshold condition determining the persistence and extinction of the disease. Furthermore, the phenomenon of Hopf bifurcation occurs when predators are introduced. The stability of limit cycle arising from a Hopf bifurcation is rigorously investigated. Finally, numerical simulations are given to show the validity of analytical results, and the comparative results of disease dynamics with and without predators.
\end{abstract}

Keywords: Vector-host diseases; Disease control; Stage structure; Hopf bifurcation; Persistence

\section{Introduction}

Host-vector diseases, such as malaria, dengue fever, tobacco mosaic virus, pine wilt disease, and so forth, are transmitted to the host population (e.g. humans and plants) by biological agents (arthropods) called vectors who carry the disease without getting themselves. Recent analysis demonstrates that the impact of environmental change and frequent occurrence of natural disasters on complex host-parasite relationships for vectorborne diseases is clear $[1,2]$. Vector-borne diseases remain a serious threat to humans, livestock, and plants, and thus the control of such diseases is of great economic and healthcare concern.

Vector control is one of the few proven ways to reduce transmission of many vectorborne diseases. The mostly adopted methods to control vectors include physical control, such as burning, clearness, burying, bednets, and so on, spraying insecticides [3], vaccination [4,5], environment control [6]. As a newly proposed method of vector-borne diseases, Sterile Insect Technique (SIT) [7] has obtained some applications in vector-borne disease control though it is still in its infancy.

One potential approach to vector control is to use biological enemies (biocontrol agents) of the vectors. Biological control shows no environmental contamination and vector resistance, less maintenance costs, and more safety compared with insecticide, environment

(c) The Author(s) 2018. This article is distributed under the terms of the Creative Commons Attribution 4.0 International License (http://creativecommons.org/licenses/by/4.0/), which permits unrestricted use, distribution, and reproduction in any medium, provided you give appropriate credit to the original author(s) and the source, provide a link to the Creative Commons license, and indicate if changes were made. 
control, and Sterile Insect Technique, respectively. Moreover, it will be conducive to ecological diversity and environment protection. Biological agents have obtained successful application in controlling a variety of host-vector diseases. For human diseases, entomogenous fungi were adopted as promising biopesticides for sleeping sickness and tick control $[8,9]$. Predators were introduced to control many diseases such as dengue fever, malaria, Lyme disease and tick disease [10-14]. Several recent studies have shown that predators have caused decline in local cases of malaria in India $[15,16]$ and dengue fever in Vietnam and Thailand $[17,18]$. In addition, biological agents were used in tree diseases such as pine wilt disease in Japan and China [19,20], as well as in crop diseases such as the cassava mosaic virus in sub-Saharan Africa and the tomato leaf curl virus in India [21, 22].

However, biological control of vectors is still only partially understood compared with biological control of herbivorous pests, which has long been established and widely applied in pest management aiming to directly reduce pest populations by pest enemies [23, 24], and many good research results of interactions between the biological agents (predators) and the pests (prey) have been stated in some papers (see, for example, [25-27] etc.). This is primarily caused by the complexity of interactions among the host, vector, and predator populations. The predators affect the spread of pathogen and the interactions between the hosts and the vectors by preying on the vectors, and the interactions between the hosts and the vectors, between the pathogen and the hosts will in turn impact the vector-predator dynamics.

Mathematical modeling provides strong support for understanding the complex dynamics of epidemic and ecological systems as well as in decision making process on disease prevention and disease control. Different mathematical models were proposed in $[28,29]$ to explore the effect of Wolbachia, which shortened the lifespan of the vector (the infected mosquito Sedes aegypti), on the transmission of dengue. Moore et al. [30] first adopted a host-vector-predator mathematical model to investigate how the predators affect the persistence or extinction of vector-borne diseases. In [31], authors considered the disease dynamics for a class of host-vector models with the effect of predators, and the nonlinear dynamics caused by predators. Okamoto and Amarasek [32] gave the comparative analysis of three classes of biocontrol agents: pathogen, predator/parasitoid, and competitor of the vector controlling diseases by reducing vector densities. However, all the above studies focused on the effect of predators on vector-borne disease control without stage structure.

In reality, individuals in a population may grow through several stages of physiology, such as immature and mature. Some epidemic models with physiology stage for the hosts have been investigated recently (see [33-38]). However, for vector-borne diseases, there are some kinds of vector-host diseases which are only spread among hosts by the immature vectors such as cysticercosis and Scrub typhus [39]. Some infectious diseases, such as malaria, dengue fever, West Nile virus, pine wilt disease [40], and so on, are spread only by the adult vectors. Consequently, to study realistically the host-vector disease transmission in a host population, we must consider the model to include stage structure for the vectors, each stage being homogeneous.

Motivated by the above discussions, in this paper we develop a host-vector-predator model with stage structure for the vectors to ask how the interactions between the vectors and the biocontrol agents indirectly reduce the prevalence of a vector-borne disease in the host population, how the predators cause the change in the host-pathogen dynamics, 
and how the stage structure impacts the disease dynamics with and without predators. The remaining part of this paper is organized as follows: In Sect. 2, we mainly formulate our model. In Sect. 3, we establish the existence and stability results of the disease and disease-free equilibria of the model, and the phenomenon of Hopf bifurcation is rigorously studied. In Sect. 4, numerical simulations are given to show the validity of our results and to compare the disease dynamics with and without predators. The paper ends with a conclusion.

The contributions of this paper can be summed as follows: (1) The coupled host-vectorpredator model with stage structure is considered, where the total vector population is divided into immature and mature subclasses. (2) We analyze the dynamics of the model by Routh-Hurwitz criteria and bifurcation theory. Theoretical results show that the introduction of predators leads to the occurrence of Hopf bifurcation. Meanwhile, the introduction of predators is greatly helpful to disease control. The effect of stage structure on disease transmission is also investigated. (3) Finally, numerical simulations are given to show the validity of analytical results, and the comparative results of disease dynamics with and without predators are presented.

\section{Model description}

To model the interactions among the host population, the vector population with stage structure, and the predator population, we divide the total vector population into two stage groups, immature vectors $M_{v}(t)$ and mature vectors $N_{v}(t)$, and assume that only mature vectors have the ability to transmit the disease to host populations. Therefore $N_{\nu}(t)$ can be divided into two subclasses, susceptible and infectious, with densities denoted by $S_{v}(t)$ and $I_{\nu}(t)$, respectively, so that $N_{v}(t)=S_{v}(t)+I_{v}(t)$. It is assumed that the virus in vectors does not cause the death of vectors and does not influence the propagation of vectors. The birth rate of the immature vector is assumed to be proportional to the density of mature vectors with proportionality parameter $b_{2}$. We assume that mature vector populations experience growing effects with rate parameter $\alpha$. We introduce the predator population for preying on the vectors which transmits the disease among hosts, and the interactions between the predators and the vectors are portrayed by Holling type II functional response, which is $h_{2} N_{v}(t) P_{2}(t) /\left(1+a_{2} N_{v}(t)\right)$, where $h_{2}$ and $a_{2}$ are the capturing rate (or the attacking rate) and the satiety rate of the predator $P_{2}(t)$, respectively, and $P_{2}(t)$ denotes the predator population of the mature vector population. The total host population is split into susceptible and infectious subclasses, with sizes denoted by $S_{h}(t)$ and $I_{h}(t)$, respectively.

Using the above assumptions, we obtain the following host-vector-predator dynamical model:

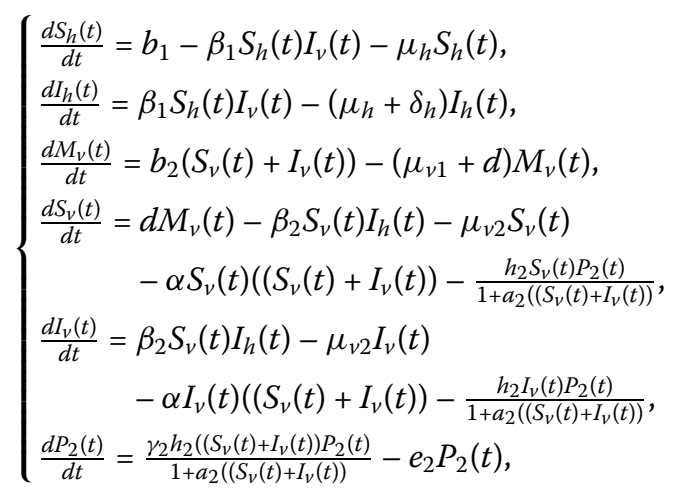


with initial conditions

$$
\begin{array}{lll}
S_{h}(0) \geq 0, & I_{h}(0) \geq 0, & M_{\nu}(0) \geq 0, \\
S_{v}(0) \geq 0, & I_{v}(0) \geq 0, & P_{2}(0) \geq 0,
\end{array}
$$

where $b_{1}$ is the recruitment rate of the host populations. $\mu_{h}$ is the natural death rate of the infected host population, $\mu_{v 1}$ and $\mu_{v 2}$ are respectively the natural death rate of the immature and mature vectors. $\delta_{h}$ is the disease-caused death rate of the infected hosts. $d$ is the conversion rate from immature vectors to mature vectors. $\beta_{1}$ and $\beta_{2}$ are respectively the rate of biting from susceptible hosts to infected vectors and susceptible vectors to infected hosts. $\gamma_{2}$ and $e_{2}$ respectively denote the conversion factor and the natural mortality of the predator populations.

Without considering the effect of predators on host-vector disease model, (1) can be reduced as follows:

$$
\left\{\begin{aligned}
\frac{d S_{h}(t)}{d t}= & b_{1}-\beta_{1} S_{h}(t) I_{v}(t)-\mu_{h} S_{h}(t) \\
\frac{d I_{h}(t)}{d t}= & \beta_{1} S_{h}(t) I_{\nu}(t)-\left(\mu_{h}+\delta_{h}\right) I_{h}(t) \\
\frac{d M_{v}(t)}{d t}= & b_{2}\left(S_{v}(t)+I_{v}(t)\right)-\left(\mu_{v 1}+d\right) M_{v}(t) \\
\frac{d S_{v}(t)}{d t}= & d M_{v}(t)-\beta_{2} S_{v}(t) I_{h}(t)-\mu_{v 2} S_{\nu}(t) \\
& -\alpha S_{v}(t)\left(\left(S_{v}(t)+I_{v}(t)\right)\right. \\
\frac{d I_{v}(t)}{d t}= & \beta_{2} S_{v}(t) I_{h}(t)-\mu_{v 2} I_{v}(t) \\
& -\alpha I_{v}(t)\left(\left(S_{v}(t)+I_{v}(t)\right)\right.
\end{aligned}\right.
$$

with initial conditions

$$
S_{h}(0) \geq 0, \quad I_{h}(0) \geq 0, \quad M_{\nu}(0) \geq 0, \quad S_{v}(0) \geq 0, \quad I_{\nu}(0) \geq 0 .
$$

\section{Dynamics of model (1)}

In this section, we mainly study the dynamics of model (1). As preliminary results, first we give the equilibria of (1).

Lemma 3.1 The equilibria for model (1) are as follows.

(i) The boundary equilibrium $E_{0}\left(b_{1} / \mu_{h}, 0,0,0,0,0\right)$ always exists.

(ii) The predator-absent disease-free equilibrium $E_{1}\left(S_{h}^{0}, 0, M_{v}^{0}, S_{v}^{0}, 0,0\right)$ exists if $\sigma>0$, where

$$
S_{h}^{0}=\frac{b_{1}}{\mu_{h}}, \quad M_{v}^{0}=\frac{b_{2} S_{v}^{0}}{\mu_{v 1}+d}, \quad S_{v}^{0}=\frac{\sigma}{\left(\mu_{v 1}+d\right) \alpha}, \quad \sigma=b_{2} d-\mu_{v 2}\left(\mu_{v 1}+d\right) .
$$

(iii) The predator-absent disease equilibrium $E_{2}\left(S_{h}^{*}, I_{h}^{*}, M_{v}^{*}, S_{v}^{*}, I_{h}^{*}, 0\right)$ exists if $\sigma>0$ and $R_{01}>1$, where

$$
\begin{aligned}
& S_{h}^{*}=\frac{b_{1}\left(\mu_{v 2}+\alpha S_{v}^{0}+\beta_{2} I_{h}^{*}\right)}{\mu_{h}\left(\mu_{v 2}+\alpha S_{v}^{0}+\beta_{2} I_{h}^{*}\right)+\beta_{1} \beta_{2} S_{v}^{0} I_{h}^{*}}, \\
& I_{h}^{*}=\frac{\mu_{h}\left(\mu_{v 2}+\alpha S_{v}^{0}\right)\left(R_{01}-1\right)}{\beta_{2}\left(\mu_{h}+\beta_{1} S_{v}^{0}\right)},
\end{aligned}
$$




$$
\begin{array}{ll}
M_{v}^{*}=\frac{b_{2} S_{v}^{0}}{\left(\mu_{v 1}+d\right)}, \quad S_{v}^{*}=\frac{\left(\mu_{v 2}+\alpha S_{v}^{0}\right) S_{v}^{0}}{\mu_{v 2}+\alpha S_{v}^{0}+\beta_{2} I_{h}^{*}}, \quad I_{v}^{*}=\frac{\beta_{2} S_{v}^{0} I_{h}^{*}}{\mu_{v 2}+\alpha S_{v}^{0}+\beta_{2} I_{h}^{*}}, \\
S_{v}^{0}=\frac{\sigma}{\left(\mu_{v 1}+d\right) \alpha}, \quad R_{01}=\frac{\beta_{1} \beta_{2} S_{h}^{0} S_{v}^{0}}{\left(\mu_{h}+\delta_{h}\right)\left(\mu_{v 2}+\alpha S_{v}^{0}\right)} .
\end{array}
$$

(iv) The predator-present disease-free equilibrium $E_{3}\left(S_{h}^{1}, 0, M_{v}^{1}, S_{v}^{1}, 0, P_{2}^{1}\right)$ exists if $\sigma>0$ and $R_{1}>1$, where $\sigma$ is given in case $(i)$, and

$$
\begin{aligned}
& S_{h}^{1}=S_{h}^{0}=\frac{b_{1}}{\mu_{h}}, \quad M_{v}^{1}=\frac{b_{2} S_{v}^{1}}{\mu_{v 1}+d}, \quad S_{v}^{1}=\frac{e_{2}}{\gamma_{2} h_{2}-a_{2} e_{2}}, \\
& P_{2}^{1}=\frac{\alpha\left(S_{v}^{0}-S_{v}^{1}\right)\left(1+a_{2} S_{v}^{1}\right)}{h_{2}}, \quad R_{1}=\frac{\sigma\left(\gamma_{2} h_{2}-a_{2} e_{2}\right)}{\left(\mu_{v 1}+d\right) \alpha e_{2}} .
\end{aligned}
$$

(v) The predator-present disease equilibrium $E_{4}\left(\hat{S}_{h}, \hat{I}_{h}, \hat{M}_{v}, \hat{S}_{v}, \hat{I}_{v}, \hat{P}_{2}\right)$ exists if $\sigma>0$, $R_{1}>1$, and $R_{02}>1$, where $\sigma$ and $R_{1}$ are given in cases (i) and (iv), respectively, and

$$
\begin{aligned}
& \hat{S}_{h}=\frac{b_{1}\left(\mu_{v 2}+\alpha S_{v}^{1}+\beta_{2} \hat{I}_{h}+\frac{h_{2} P_{2}^{1}}{1+a_{2} S_{v}^{1}}\right)}{\mu_{h}\left(\mu_{v 2}+\alpha S_{v}^{1}+\beta_{2} \hat{I}_{h}+\frac{h_{2} P_{2}^{1}}{1+a_{2} S_{v}^{1}}\right)+\beta_{1} \beta_{2} S_{v}^{1} \hat{I}_{h}}, \\
& \hat{I}_{h}=\frac{\mu_{h}\left(\mu_{v 2}+\alpha S_{v}^{1}+\frac{h_{2} P_{2}^{1}}{1+a_{2} S_{v}^{1}}\right)\left(R_{02}-1\right)}{\beta_{2}\left(\mu_{h}+\beta_{1} S_{v}^{1}\right)}, \\
& \hat{M}_{v}=\frac{b_{2} S_{v}^{1}}{\left(\mu_{v 1}+d\right)}, \quad \hat{S}_{v}=\frac{\left(\mu_{v 2}+\alpha S_{v}^{1}+\frac{h_{2} P_{2}^{1}}{1+a_{2} S_{v}^{1}}\right) S_{v}^{1}}{\mu_{v}+\alpha S_{v}^{1}+\beta_{2} \hat{I}_{h}+\frac{h_{2} P_{2}^{1}}{1+a_{2} S_{v}^{1}}}, \\
& \hat{I}_{v}=\frac{\beta_{2} S_{v}^{1} \hat{I}_{h}}{\mu_{v 2}+\alpha S_{v}^{1}+\beta_{2} \hat{I}_{h}+\frac{h_{2} P_{2}^{1}}{1+a_{2} S_{v}^{1}}}, \quad S_{v}^{1}=\frac{e_{2}}{\gamma_{2} h_{2}-a_{2} e_{2}}, \\
& \hat{P}_{2}=P_{2}^{1}=\frac{\alpha\left(S_{v}^{0}-S_{v}^{1}\right)\left(1+a_{2} S_{v}^{1}\right)}{h_{2}}, \\
& R_{02}=\frac{\beta_{1} \beta_{2} S_{h}^{0} S_{v}^{1}}{\left(\mu_{h}+\delta_{h}\right)\left(\mu_{v 2}+\alpha S_{v}^{0}\right)} .
\end{aligned}
$$

The equilibria $E_{i}(i=0,1,2,3,4)$ of Lemma 3.1 can be obtained by direct computation. Here we omit it.

According to the next generation matrix proposed in [41, 42], $R_{01}$ and $R_{02}$ given in Lemma 3.1 are the basic reproduction numbers of system (1) and system (2), respectively. It is clear that $R_{02}<R_{01}$ if $R_{1}>1$ (where the condition $R_{1}>1$ ensures $S_{v}^{0}>S_{v}^{1}$, therefore the equilibrium density of the predator is larger than zero). That is, the basic reproduction number has been lessened by introducing the predators.

\subsection{Local stability and existence of Hopf bifurcation for system (1)}

In this subsection, we shall investigate the local properties of the equilibria and Hopf bifurcation for system (1).

Theorem 3.1 For the predator-absent equilibria $E_{0}, E_{1}$, and $E_{2}$ of system (1), we have:

(i) The equilibrium $E_{0}$ is a saddle point, which is unstable. 
(ii) If $R_{1}<1, \gamma_{2} h_{2}-a_{2} e_{2}>0$, and $R_{01}<1$, then the predator-absent disease-free equilibrium $E_{1}$ is locally asymptotically stable.

(iii) If $R_{1}<1, \gamma_{2} h_{2}-a_{2} e_{2}>0$, and $R_{01}>1$, then the predator-absent disease equilibrium $E_{2}$ is locally asymptotically stable.

Here $E_{0}, E_{1}$, and $E_{2}$ are given in Lemma 3.1.

Proof By some mathematical deductions and using the Routh-Hurwitz criteria, we can obtain the proof of Theorem 3.1. Here we omit it.

By Theorem 3.1, we obtain the following corollary.

Corollary 3.1 The equilibria of system (2) are as follows.

(i) The boundary equilibrium $E_{0}\left(b_{1} / \mu_{h}, 0,0,0,0\right)$ is a saddle point, which is unstable.

(ii) If $R_{01}<1$, then the disease-free equilibrium $E_{1}\left(S_{h}^{0}, 0, M_{v}^{0}, S_{v}^{0}, 0\right)$ is locally asymptotically stable.

(iii) If $R_{01}>1$, then the disease equilibrium $E_{2}\left(S_{h}^{*}, I_{h}^{*}, M_{v}^{*}, S_{v}^{*}, I_{h}^{*}\right)$ is locally asymptotically stable,

where $S_{h}^{0}, M_{v}^{0}, S_{v}^{0}, S_{h}^{*}, I_{h}^{*}, M_{v}^{*}, S_{v}^{*}$, and $I_{h}^{*}$ are given in Lemma 3.1.

From Corollary 3.1, the basic reproduction number $R_{01}$ of system (2) is the threshold to decide the disease persistence and extinction without predators.

When $R_{1}>1$, system (1) has the predator-present equilibria $E_{3}$ and $E_{4}$ given in Lemma 3.1. In the following, we will give the stability results of $E_{3}$ and $E_{4}$.

Theorem 3.2 For the predator-present equilibria $E_{3}$ and $E_{4}$ of system (1), we have:

(i) The predator-present disease-free equilibrium $E_{3}$ is locally asymptotically stable if $R_{02}<1$ and $C_{1} C_{2}-C_{3}>0$, in which case the vector-host disease can be eradicated when a predator population $P_{2}$ is introduced. When $C_{1} C_{2}-C_{3}=0$ and $R_{02}<1$, then system (1) undergoes a Hopf bifurcation at $E_{3}$, in which case, despite oscillations occurring between the predator and the vector, predation can eliminate the pathogen and the vector population is greater than zero.

(ii) The predator-present disease equilibrium $E_{4}$ is locally asymptotically stable if $R_{02}>1$ and $C_{1} C_{2}-C_{3}>0$, in which case the vector-borne diseases persist though the predators are introduced in the system. When $C_{1} C_{2}-C_{3}=0$ and $R_{02}>1$, then system (1) undergoes a Hopf bifurcation at $E_{4}$, in which case predation causes oscillations among host, vector, and predator populations, and the disease cannot be eliminated though predators are introduced in the system.

Here

$$
\begin{aligned}
& C_{1}=\mu_{v 1}+\mu_{v 2}+d+2 \alpha S_{v}^{1}+G_{1}, \\
& C_{2}=G_{2} G_{3}+\left(\mu_{v 1}+d\right)\left(\mu_{v 2}+2 \alpha S_{v}^{1}+G_{1}\right)-b_{2} d, \\
& C_{3}=\left(\mu_{v 1}+d\right) G_{2} G_{3}, \\
& G_{1}=\frac{h_{2} P_{2}^{1}}{\left(1+a_{2} S_{v}^{1}\right)^{2}},
\end{aligned}
$$




$$
\begin{aligned}
G_{2} & =\frac{h_{2} S_{v}^{1}}{1+a_{2} S_{v}^{1}}, \\
G_{3} & =\frac{\gamma_{2} h_{2} P_{2}^{1}}{\left(1+a_{2} S_{v}^{1}\right)^{2}},
\end{aligned}
$$

where $S_{v}^{1}$ and $P_{2}^{1}$ are given in Lemma 3.1.

Proof (i) By some mathematical deductions and rearrangement, the characteristic polynomial corresponding to the disease-free equilibrium $E_{3}$ can be rewritten as

$$
f(\lambda)=\left(\lambda+\mu_{h}\right)\left(\lambda^{2}+B_{1} \lambda+B_{2}\right)\left(\lambda^{3}+C_{1} \lambda^{2}+C_{2} \lambda+C_{3}\right)=0,
$$

where

$$
\begin{aligned}
& B_{1}=\mu_{h}+\delta_{h}+\mu_{v 2}+\alpha S_{v}^{1}+\frac{h_{2} P_{2}^{1}}{1+a_{2} S_{v}^{1}}, \\
& B_{2}=\left(\mu_{h}+\delta_{h}\right)\left(1-R_{02}\right)\left[\mu_{v 2}+\alpha S_{v}^{1}+\frac{h_{2} P_{2}^{1}}{1+a_{2} S_{v}^{1}}\right],
\end{aligned}
$$

$C_{1}, C_{2}$, and $C_{3}$ are given in Theorem 3.2.

Obviously, Eq. (9) has a real root $\lambda_{1}=-\mu_{h}$, two roots $\lambda_{2,3}$ with negative real parts if $R_{02}<1$, and the other three eigenvalues can be obtained by solving

$$
\lambda^{3}+C_{1} \lambda^{2}+C_{2} \lambda+C_{3}=0
$$

For Eq. (10), three eigenvalues have negative real parts if they satisfy the Routh-Hurwitz criteria, such that $C_{i}>0, i=1,2,3$ and $C_{1} C_{2}-C_{3}>0$. From the above expressions, we see that $C_{1}>0, C_{3}>0$, and if $C_{1} C_{2}-C_{3}>0$, then we have $C_{2}>0$. Thus, $E_{3}$ is locally asymptotically stable if $R_{02}<1$ and $C_{1} C_{2}-C_{3}>0$.

Choose $h_{2}$ as a bifurcation parameter and let $h^{*}$ be the solution of equation $C_{1} C_{2}-C_{3}=$ 0 , that is, $C_{1}\left(h_{2}^{*}\right) C_{2}\left(h_{2}^{*}\right)-C_{3}\left(h_{2}^{*}\right)=0$, then Eq. (10) can be rewritten into

$$
\left(\lambda^{2}+C_{2}\right)\left(\lambda+C_{1}\right)=0
$$

which has three roots

$$
\lambda_{1}=+i \sqrt{C_{2}}, \quad \lambda_{2}=-i \sqrt{C_{2}}, \quad \lambda_{3}=-C_{1} .
$$

For $h_{2} \in\left(h_{2}^{*}-\delta, h_{2}^{*}+\delta\right)(\delta>0)$, the roots are in general of following form:

$$
\lambda_{1}\left(h_{2}\right)=w_{1}\left(h_{2}\right)+i w_{2}\left(h_{2}\right), \quad \lambda_{2}\left(h_{2}\right)=w_{1}\left(h_{2}\right)-i w_{2}\left(h_{2}\right), \quad \lambda_{3}\left(h_{2}\right)=-C_{1}\left(h_{2}\right) .
$$

Now we verify the transversality condition

$$
\operatorname{Re}\left[\frac{d \lambda_{i}}{d h_{2}}\right]_{\mid h_{2}=h_{2}^{*}} \neq 0, \quad i=1,2,3
$$


Substituting $\lambda_{1}\left(h_{2}\right)=w_{1}\left(h_{2}\right)+i w_{2}\left(h_{2}\right)$ in (11) and calculating the derivative, we get

$$
\begin{aligned}
& E_{1}\left(h_{2}\right) w_{1}^{\prime}\left(h_{2}\right)-E_{2}\left(h_{2}\right) w_{2}^{\prime}\left(h_{2}\right)+F\left(h_{2}\right)=0, \\
& E_{2}\left(h_{2}\right) w_{1}^{\prime}\left(h_{2}\right)+E_{1}\left(h_{2}\right) w_{2}^{\prime}\left(h_{2}\right)+G\left(h_{2}\right)=0,
\end{aligned}
$$

where

$$
\begin{aligned}
& E_{1}\left(h_{2}\right)=3 w_{1}^{2}\left(h_{2}\right)+2 C_{1}\left(h_{2}\right) w_{1}\left(h_{2}\right)+C_{2}\left(h_{2}\right)-3 w_{2}^{2}\left(h_{2}\right), \\
& E_{2}\left(h_{2}\right)=6 w_{1}\left(h_{2}\right) w_{2}\left(h_{2}\right)+2 C_{1}\left(h_{2}\right) w_{2}\left(h_{2}\right), \\
& F\left(h_{2}\right)=w_{1}^{2}\left(h_{2}\right) C_{1}^{\prime}\left(h_{2}\right)+w_{1}\left(h_{2}\right) C_{2}^{\prime}\left(h_{2}\right)+C_{3}^{\prime}\left(h_{2}\right)-w_{2}^{2}\left(h_{2}\right) C_{1}^{\prime}\left(h_{2}\right), \\
& G\left(h_{2}\right)=2 w_{1}\left(h_{2}\right) w_{2}\left(h_{2}\right) C_{1}^{\prime}\left(h_{2}\right)+w_{2}\left(h_{2}\right) C_{2}^{\prime}\left(h_{2}\right) .
\end{aligned}
$$

Since $E_{1}\left(h_{2}^{*}\right) F\left(h_{2}^{*}\right)+E_{2}\left(h_{2}^{*}\right) G\left(h_{2}^{*}\right) \neq 0$, thus we have

$$
\operatorname{Re}\left[\frac{d \lambda_{i}}{d h_{2}}\right]_{\mid h_{2}=h_{2}^{*}}=w_{1}^{\prime}\left(h_{2}\right)_{\mid h_{2}=h_{2}^{*}}=\frac{E_{1}\left(h_{2}^{*}\right) F\left(h_{2}^{*}\right)+E_{2}\left(h_{2}^{*}\right) G\left(h_{2}^{*}\right)}{E_{1}^{2}+E_{2}^{2}} \neq 0, \quad i=1,2,
$$

and

$$
\operatorname{Re}\left[\frac{d \lambda_{3}}{d h_{2}}\right]_{h_{2}=h_{2}^{*}}=-\operatorname{Re}\left[\frac{d C_{1}\left(h_{2}\right)}{d h_{2}}\right]_{h_{2}=h_{2}^{*}}=-\frac{\left(1+a e_{2} S_{v}^{0}\right) e_{2}}{\alpha \gamma_{2}\left(h_{2}^{*}\right)^{2}} \neq 0 .
$$

Here $S_{v}^{0}$ is given in Lemma 3.1.

Thus, system (1) undergoes a Hopf bifurcation at $E_{3}$ when $R_{1}>1, R_{02}<1$, and $h_{2}$ passes through the critical value $h_{2}^{*}$ such that $C_{1}\left(h_{2}^{*}\right) C_{2}\left(h_{2}^{*}\right)-C_{3}\left(h_{2}^{*}\right)=0$.

(ii) By some mathematical deduction and rearrangement, the characteristic polynomial corresponding to $E_{4}$ can be rewritten as

$$
\left(\lambda^{3}+A_{1} \lambda^{2}+A_{2} \lambda+A_{3}\right)\left(\lambda^{3}+C_{1} \lambda^{2}+C_{2} \lambda+C_{3}\right)=0
$$

where $C_{1}, C_{2}, C_{3}$ are given in (3), (4), and (5), respectively, and

$$
\begin{aligned}
A_{1}= & \mu_{h}+\beta_{1} \hat{I}_{v}+\mu_{h}+\delta_{h}+\mu_{v 2}+\beta_{2} \hat{I}_{h}+\alpha S_{v}^{1}+\frac{h_{2} \hat{P}_{2}}{1+a_{2} S_{v}^{1}}>0, \\
A_{2}= & \left(\mu_{h}+\beta_{1} \hat{I}_{v}\right)\left(\mu_{h}+\delta_{h}\right)+\left(\mu_{h}+\beta_{1} \hat{I}_{v}\right)\left(\mu_{v 2}+\beta_{2} \hat{I}_{h}+\alpha S_{v}^{1}+\frac{h_{2} \hat{P}_{2}}{1+a_{2} S_{v}^{1}}\right) \\
& +\left(\mu_{h}+\delta_{h}\right)\left(\mu_{v 2}+\beta_{2} \hat{I}_{h}+\alpha S_{v}^{1}+\frac{h_{2} \hat{P}_{2}}{1+a_{2} S_{v}^{1}}\right)-\beta_{1} \beta_{2} \hat{S}_{h} \hat{S}_{v} \\
= & \left(\mu_{h}+\beta_{1} \hat{I}_{v}\right)\left(\mu_{h}+\delta_{h}\right)+\left(\mu_{h}+\beta_{1} \hat{I}_{v}\right)\left(\mu_{v 2}+\beta_{2} \hat{I}_{h}+\alpha S_{v}^{1}+\frac{h_{2} \hat{P}_{2}}{1+a_{2} S_{v}^{1}}\right) \\
& +\left(\mu_{h}+\delta_{h}\right)\left(\mu_{v 2}+\beta_{2} \hat{I}_{h}+\alpha S_{v}^{1}+\frac{h_{2} \hat{P}_{2}}{1+a_{2} S_{v}^{1}}\right)-\left(\mu_{h}+\delta_{h}\right)\left(\mu_{v 2}+\alpha S_{v}^{1}+\frac{h_{2} \hat{P}_{2}}{1+a_{2} S_{v}^{1}}\right) \\
> & \left(\mu_{h}+\beta_{1} \hat{I}_{v}\right)\left(\mu_{h}+\delta_{h}+\mu_{v 2}+\beta_{2} \hat{I}_{h}+\alpha S_{v}^{1}+\frac{h_{2} \hat{P}_{2}}{1+a_{2} S_{v}^{1}}\right)>0,
\end{aligned}
$$




$$
\begin{aligned}
A_{3}= & \left(\mu_{h}+\beta_{1} \hat{I}_{v}\right)\left(\mu_{h}+\delta_{h}\right)\left(\mu_{v 2}+\beta_{2} \hat{I}_{h}+\alpha S_{v}^{1}+\frac{h_{2} \hat{P}_{2}}{1+a_{2} S_{v}^{1}}\right)-\mu_{h} \beta_{1} \beta_{2} \hat{S}_{h} \hat{S}_{v} \\
= & \left(\mu_{h}+\beta_{1} \hat{I}_{v}\right)\left(\mu_{h}+\delta_{h}\right)\left(\mu_{v 2}+\beta_{2} \hat{I}_{h}+\alpha S_{v}^{1}+\frac{h_{2} \hat{P}_{2}}{1+a_{2} S_{v}^{1}}\right) \\
& -\mu_{h}\left(\mu_{h}+\delta_{h}\right)\left(\mu_{v 2}+\alpha S_{v}^{1}+\frac{h_{2} \hat{P}_{2}}{1+a_{2} S_{v}^{1}}\right)>0,
\end{aligned}
$$

where $\hat{I}_{v}, \hat{I}_{h}, \hat{S}_{v}, \hat{S}_{h}, \hat{P}_{2}$, and $S_{v}^{1}$ are given in Lemma 3.1, and

$$
\begin{aligned}
A_{1} A_{2}-A_{3}> & \left(\mu_{h}+\beta_{1} \hat{I}_{v}+\mu_{h}+\delta_{h}+\mu_{v 2}+\beta_{2} \hat{I}_{h}+\alpha S_{v}^{1}+\frac{h_{2} \hat{P}_{2}}{1+a_{2} S_{v}^{1}}\right)\left(\mu_{h}+\beta_{1} \hat{I}_{v}\right) \\
& \cdot\left[\left(\mu_{h}+\delta_{h}\right)+\left(\mu_{v 2}+\beta_{2} \hat{I}_{h}+\alpha S_{v}^{1}+\frac{h_{2} \hat{P}_{2}}{1+a_{2} S_{v}^{1}}\right)\right]-\left(\mu_{h}+\beta_{1} \hat{I}_{v}\right)\left(\mu_{h}+\delta_{h}\right) \\
& \cdot\left(\mu_{v 2}+\beta_{2} \hat{I}_{h}+\alpha S_{v}^{1}+\frac{h_{2} \hat{P}_{2}}{1+a_{2} S_{v}^{1}}\right)>0 .
\end{aligned}
$$

Therefore, by the Routh-Hurwitz criteria, equation $\left(\lambda^{3}+A_{1} \lambda^{2}+A_{2} \lambda+A_{3}\right)=0$ has three roots with negative real parts. For the equation $\lambda^{3}+C_{1} \lambda^{2}+C_{2} \lambda+C_{3}=0$, by the analysis results of disease-free $E_{3}$ in Theorem 3.2, we have that the disease equilibrium $E_{4}$ is locally asymptotically stable if $R_{02}>1$ and $C_{1} C_{2}-C_{3}>0$, and if $R_{02}>1$, then system (1) undergoes a Hopf bifurcation at the disease equilibrium $E_{4}$ when $h_{2}$ passes through the critical value $h_{2}^{*}$ such that $C_{1}\left(h_{2}^{*}\right) C_{2}\left(h_{2}^{*}\right)-C_{3}\left(h_{2}^{*}\right)=0$.

Hence the proof of Theorem 3.2.

Remark 1 From Theorem 3.2, we find that the introduction of predation into vectors results in the occurrence of Hopf bifurcation for system (1), and the condition $C_{1} C_{2}-C_{3}=0$ is the threshold to determine the existence of Hopf bifurcation. On the other hand, when $R_{01}>1$ then the disease persists. By introducing predators to the vectors, it is clear that the basic reproduction number $R_{02}$ takes the role of threshold determining the persistence and extinction of the disease. If $R_{02}>1$, then the vector-borne diseases will persist. If $R_{02}<1$, then the vector-borne diseases will tend to die out. That is to say, reducing the value of $R_{2}$ will promote the positive effect of predators on disease control. In the next numerical simulation, we will further analyze the relationship between some predator parameters and the value of $R_{02}$, and present some simulation figures to show the positive role of predators in disease control.

\subsection{Direction and stability of limit cycle}

In this subsection, we study the stability of a limit cycle caused by the predator preying on the mature vector population. Before giving the main results, we first discuss the following subsystem:

$$
\left\{\begin{array}{l}
\frac{d M_{v}(t)}{d t}=b_{2} N_{v}(t)-\left(\mu_{v 1}+d\right) M_{v}(t) \\
\frac{d N_{v}(t)}{d t}=d M_{v}(t)-\mu_{v 2} N_{v}(t)-\alpha N_{v}^{2}(t)-\frac{h_{2} N_{v}(t) P_{2}(t)}{1+a_{2} N_{v}(t)} \\
\frac{d P_{2}(t)}{d t}=\frac{\gamma_{2} h_{2} N_{v}(t) P_{2}(t)}{1+a_{2} N_{v}(t)}-e_{2} P_{2}(t)
\end{array}\right.
$$


where $N_{v}(t)$ is the total mature vector population density at time $t$, that is, $N_{v}(t)=S_{v}(t)+$ $I_{\nu}(t)$.

For system (16), there always exist a predator-free equilibrium $E_{0}\left(M_{v}^{0}, N_{v}^{0}, 0\right)$ and a predator-present equilibrium $E_{1}\left(\tilde{M}_{v}, \tilde{N}_{v}, \tilde{P}_{2}\right)$ if $R_{1}>1$, where $N_{v}^{0}=S_{v}^{0}, \tilde{M}_{v}=M_{v}^{1}, \tilde{N}_{v}=S_{v}^{1}$, $\tilde{P}_{2}=P_{2}^{1}$, and $M_{v}^{0}, S_{v}^{0}, M_{v}^{1}, S_{v}^{1}, P_{2}^{1}$ are given in Lemma 3.1.

It is easy to find that the equilibrium $E_{0}$ of system (16) is locally asymptotically stable if $R_{1}<1$ and unstable if $R_{1}>1$. The characteristic polynomial at $E_{1}$ of system (16) is

$$
\lambda^{3}+C_{1} \lambda^{2}+C_{2} \lambda+C_{3}=0
$$

Then, by Theorem 3.2, we have the following results of the local stability and existence of Hopf bifurcation for system (16).

Corollary 3.2 The local stability and existence of Hopf bifurcation of system (16) are as follows:

(i) $E_{0}\left(M_{v}^{0}, N_{v}^{0}, 0\right)$ is locally asymptotically stable if $R_{1}<1$ and unstable if $R_{1}>1$.

(ii) $E_{1}\left(\tilde{M}_{v}, \tilde{N}_{v}, \tilde{P}_{2}\right)$ is locally asymptotically stable if $R_{1}>1$ and $C_{1} C_{2}-C_{3}>0$.

(iii) When $h_{2}$ passes through the critical value $h_{2}^{*}$, which satisfies $C_{1}\left(h_{2}^{*}\right) C_{2}\left(h_{2}^{*}\right)-C_{3}\left(h_{2}^{*}\right)=0$, system (16) undergoes a Hopf bifurcation at the equilibrium $E_{1}\left(\tilde{M}_{v}, \tilde{N}_{v}, \tilde{P}_{2}\right)$,

where $C_{1}, C_{2}, C_{3}$ are given in (3), (4), and (5), respectively.

In the following, we apply the center manifold theorem to study the stability of the limit cycle arising from Hopf bifurcation. For convenience, we first translate the origin of the coordinate system to the equilibrium $E_{1}\left(\tilde{M}_{v}, \tilde{N}_{v}, \tilde{P}_{2}\right)$ by writing

$$
\bar{M}_{v}=M_{v}-\tilde{M}_{v}, \quad \bar{I}_{v}=I_{v}-\tilde{I}_{v}, \quad \bar{P}_{2}=P_{2}-\tilde{P}_{2}
$$

then from (16) we have

$$
\frac{d}{d t}\left(\begin{array}{c}
\bar{M}_{v} \\
\bar{N}_{v} \\
\bar{P}_{2}
\end{array}\right)=\tilde{J}\left(\begin{array}{c}
\bar{M}_{v} \\
\bar{N}_{v} \\
\bar{P}_{2}
\end{array}\right)+\left(\begin{array}{c}
\varphi_{1} \\
\varphi_{2} \\
\varphi_{3}
\end{array}\right)
$$

where

$$
\tilde{J}=\left(\begin{array}{ccc}
-\left(\mu_{v 1}+d\right) & b_{2} & 0 \\
d & -\left(\mu_{v 2}+2 \alpha S_{v}^{1}+G_{1}\right) & -G_{2} \\
0 & G_{3} & 0
\end{array}\right)
$$

and the nonlinear terms $\varphi_{1}, \varphi_{2}$, and $\varphi_{3}$ are given by

$$
\varphi_{1}=0, \quad \varphi_{2}=-G_{4} \bar{N}_{v}^{2}-G_{5} \bar{N}_{v} \bar{P}_{2}, \quad \varphi_{3}=-G_{6} \bar{N}_{v} \bar{P}_{2},
$$

where

$$
G_{4}=\alpha+\frac{a_{2} h_{2} P_{2}^{1}}{\left(1+a_{2} S_{v}^{1}\right)^{3}}, \quad G_{5}=\frac{h_{2}}{\left(1+a_{2} S_{v}^{1}\right)^{2}}, \quad G_{6}=\frac{a_{2} h_{2} \gamma_{2} P_{2}^{1}}{\left(1+a_{2} S_{v}^{1}\right)^{3}} .
$$


When the Hopf bifurcation characteristic equation holds, then the eigenvalues of $\tilde{J}$ are $\lambda_{1,2}= \pm i \eta, \lambda_{3}=\theta$, where $\theta=-C_{1}, \eta^{2}=C_{2}=G_{2} G_{3}+\left(\mu_{v 1}+d\right)\left(\mu_{v 2}+2 \alpha S_{v}^{1}+G_{1}\right)-b_{2} d$.

If the eigenvectors of $\tilde{J}$ associated with $\lambda_{1,2}$ are $W_{1} \pm i W_{2}$, and the eigenvector corresponding to $\lambda_{3}$ is $W_{3}$, then it can be shown that the matrix $P=\left(W_{2}, W_{1}, W_{3}\right)$ is nonsingular. Furthermore,

$$
P^{-1} \tilde{J} P=\left(\begin{array}{ccc}
0 & -\eta & 0 \\
\eta & 0 & 0 \\
0 & 0 & \theta
\end{array}\right)
$$

and $P$ is given by

$$
P=\left[p_{i j}\right] \quad(i, j=1,2,3)
$$

where

$$
\begin{aligned}
& p_{11}=0, \quad p_{12}=1, \quad p_{13}=\frac{b_{2}}{\mu_{v 1}+d+\theta}, \\
& p_{21}=\frac{\eta}{b_{2}}, \quad p_{22}=\frac{\mu_{v 1}+d}{b_{2}}, \\
& p_{23}=1, \quad p_{31}=-\frac{G_{3}\left(\mu_{v 1}+d\right)}{b_{2} \eta}, \quad p_{32}=\frac{G_{3}}{b_{2}}, \quad p_{33}=\frac{G_{3}}{\theta},
\end{aligned}
$$

and

$$
Q=P^{-1}=\Delta\left[q_{i j}\right] \quad(i, j=1,2,3),
$$

where $\Delta=\operatorname{det} P^{-1}$ and

$$
\begin{array}{lll}
q_{11}=p_{22} p_{33}-p_{23} p_{32}, & q_{12}=p_{32} p_{13}-p_{12} p_{33}, & q_{13}=p_{12} p_{23}-p_{13} p_{22}, \\
q_{21}=p_{31} p_{23}-p_{21} p_{33}, & q_{22}=p_{11} p_{11}-p_{31} p_{13}, & q_{23}=p_{21} p_{13}-p_{11} p_{23}, \\
q_{31}=p_{21} p_{32}-p_{22} p_{31}, & q_{32}=p_{31} p_{12}-p_{11} p_{32}, & q_{33}=p_{11} p_{22}-p_{12} p_{21} .
\end{array}
$$

Let the linear transformation

$$
\bar{Y}=P W,
$$

where $\bar{Y}=\left(\bar{M}_{v}, \bar{N}_{v}, \bar{P}_{2}\right)^{T}$ and $W=\left(x_{1}, x_{2}, x_{3}\right)^{T}$, then we have

$$
W=P^{-1} \bar{Y},
$$

where $P$ is given by (21).

Substituting (23) into (17), we get

$$
\frac{d(P W)}{d t}=\tilde{J} P W+H(P W)
$$


where $H(P W)=H(\bar{Y})=\left(\varphi_{1}, \varphi_{2}, \varphi_{3}\right)^{T}$, which implies that

$$
\frac{d W}{d t}=\left(P^{-1} \tilde{J} P\right) W+P^{-1} H(P W)
$$

where $P^{-1} \tilde{J} P$ is a constant matrix given by (20).

Now system (24) can be written as

$$
\left\{\begin{array}{l}
\dot{x}=A x+F_{1}(x, y), \\
\dot{y}=B y+F_{2}(x, y)
\end{array}\right.
$$

where $x=\left(x_{1}, x_{2}\right)^{T}, y=\left(x_{3}\right), A$ and $B$ are the constant matrices given by

$$
A=\left(\begin{array}{cc}
0 & -\eta \\
\eta & 0
\end{array}\right), \quad B=(\theta)
$$

and $F_{1}, F_{2}$ are functions of $C^{2}$.

We can write system (24) as

$$
\frac{d}{d t}\left(\begin{array}{l}
x_{1} \\
x_{2} \\
x_{3}
\end{array}\right)=\left(\begin{array}{ccc}
0 & -\eta & 0 \\
\eta & 0 & 0 \\
0 & 0 & \theta
\end{array}\right)\left(\begin{array}{l}
x_{1} \\
x_{2} \\
x_{3}
\end{array}\right)+Q\left(\begin{array}{l}
\varphi_{1} \\
\varphi_{2} \\
\varphi_{3}
\end{array}\right)
$$

From (27) we have that

$$
\frac{d x_{3}}{d t}=\theta x_{3}+\Delta\left(q_{31} \varphi_{1}+q_{32} \varphi_{2}+q_{33} \varphi_{3}\right)
$$

To prove the stability of limit cycle, we give the following two lemmas.

Lemma 3.2 ([43]) System (27) has a local center manifold $y=\psi(x), K<\delta$, where $\psi$ is in $C^{2}$. The function $\psi(x)$ can be approximated arbitrarily closed as a Taylor series as proved by the following theorem.

Lemma 3.3 ([43]) Let $\phi: S^{n} \rightarrow S^{m}$ be $C^{1}$ in a neighborhood of origin, $\phi(0)=0, \phi^{\prime}(0)=0$, and $M \phi(x)=O\left(|x|^{p}\right)$ as $x \rightarrow \infty$, then $\psi(x)=\phi(x)+O\left(|x|^{p}\right)$ as $x \rightarrow \infty$, where $M \phi(x)=$ $\phi^{\prime}(x)\left[B x+F_{2}(x, \phi(x))\right]-A \phi(x)-F_{1}(x, \phi(x))$ and $p>1$.

Hence, by Lemma 3.3, the center manifold up to a quadratic approximation can be described by

$$
x_{3}=\psi\left(x_{1}, x_{2}\right)=\frac{1}{2}\left(a_{11} x_{1}^{2}+2 a_{12} x_{1} x_{2}+a_{22} x_{2}^{2}\right) .
$$

Then it follows that

$$
\frac{d x_{3}}{d t}=\left(\begin{array}{ll}
a_{11} x_{1}+a_{12} x_{2} & a_{12} x_{1}+a_{22} x_{2}
\end{array}\right)\left(\begin{array}{cc}
0 & -\eta \\
\eta & 0
\end{array}\right)\left(\begin{array}{l}
x_{1} \\
x_{2}
\end{array}\right) .
$$


On the other hand, by (28) and (29) we have

$$
\frac{d x_{3}}{d t}=\frac{\theta}{2}\left(a_{11} x_{1}^{2}+2 a_{12} x_{1} x_{2}+a_{22} x_{2}^{2}\right)+\Delta\left(q_{31} \varphi_{1}+q_{32} \varphi_{2}+q_{33} \varphi_{3}\right)
$$

From (23), we have

$$
\begin{aligned}
& \bar{M}_{v}=p_{11} x_{1}+p_{12} x_{2}+p_{13} x_{3}, \quad \bar{N}_{v}=p_{21} x_{1}+p_{22} x_{2}+p_{23} x_{3}, \\
& \bar{P}_{2}=p_{31} x_{1}+p_{32} x_{2}+p_{33} x_{3} .
\end{aligned}
$$

By substituting $\bar{M}_{v}, \bar{N}_{v}$, and $\bar{P}_{2}$ from (32) into Eq. (19) and rearranging, we then obtain $\varphi_{1}, \varphi_{2}$, and $\varphi_{3}$ as follows:

$$
\begin{aligned}
\varphi_{1}= & 0, \\
\varphi_{2}= & -G_{4}\left(p_{21} x_{1}+p_{22} x_{2}+p_{23} x_{3}\right)^{2}-G_{5}\left(p_{21} x_{1}+p_{22} x_{2}+p_{23} x_{3}\right) \\
& \cdot\left(p_{31} x_{1}+p_{32} x_{2}+p_{33} x_{3}\right), \\
= & -G_{4}\left(p_{21} x_{1}+p_{22} x_{2}\right)^{2}-G_{4} p_{23} \Lambda\left(p_{21} x_{1}+p_{22} x_{2}\right) \\
& -G_{5}\left(p_{21} x_{1}+p_{22} x_{2}\right)\left(p_{31} x_{1}+p_{32} x_{2}\right) \\
& -\frac{1}{2} G_{5} p_{33} \Lambda\left(p_{21} x_{1}+p_{22} x_{2}\right)-\frac{1}{2} G_{5} p_{23} \Lambda\left(p_{31} x_{1}+p_{32} x_{2}\right), \\
\varphi_{3}= & -G_{6} \bar{N}_{v} \bar{P}_{2} \\
= & -G_{6}\left(p_{21} x_{1}+p_{22} x_{2}\right)\left(p_{31} x_{1}+p_{32} x_{2}\right) \\
& -\frac{1}{2} G_{6} p_{33} \Lambda\left(p_{21} x_{1}+p_{22} x_{2}\right)-\frac{1}{2} G_{6} p_{23} \Lambda\left(p_{31} x_{1}+p_{32} x_{2}\right),
\end{aligned}
$$

where $\Lambda=a_{11} x_{1}^{2}+2 a_{12} x_{1} x_{2}+a_{22} x_{2}^{2}$.

Now it follows from (30) and (31) that

$$
\begin{aligned}
\frac{d x_{3}}{d t}= & \left(\begin{array}{ll}
a_{11} x_{1}+a_{12} x_{2} & \left.a_{12} x_{1}+a_{22} x_{2}\right)
\end{array}\right)\left(\begin{array}{cc}
0 & -\eta \\
\eta & 0
\end{array}\right)\left(\begin{array}{l}
x_{1} \\
x_{2}
\end{array}\right) \\
= & \frac{\theta}{2}\left(a_{11} x_{1}^{2}+2 a_{12} x_{1} x_{2}+a_{22} x_{2}^{2}\right)+\Delta\left(q_{31} \varphi_{1}+q_{32} \varphi_{2}+q_{33} \varphi_{3}\right) \\
= & x_{1}^{2}\left[\frac{\theta}{2} a_{11}-\Delta\left(q_{32}\left(G_{4} p_{21}^{2}+G_{5} p_{21} p_{31}\right)+q_{33} G_{6} p_{21} p_{31}\right)\right]+x_{1} x_{2} \\
& \cdot\left[\theta a_{12}-\Delta\left(q_{32}\left(2 G_{4} p_{21} p_{22}+G_{5}\left(p_{21} p_{32}+p_{22} p_{31}\right)\right)+q_{33} G_{6}\left(p_{21} p_{32}+p_{22} p_{31}\right)\right)\right] \\
& +x_{2}^{2}\left[\frac{\theta}{2} a_{22}-\Delta\left(q_{32}\left(G_{4} p_{22}^{2}+G_{5} p_{22} p_{32}\right)+q_{33} G_{6} p_{22} p_{32}\right)\right]+o\left(\rho^{2}\right)
\end{aligned}
$$

where $o\left(\rho^{2}\right)$ represents higher order term when $\rho^{2} \rightarrow 0, \rho=\sqrt{x^{2}+y^{2}}$.

Comparing the coefficient of $x_{1}^{2}, x_{1} x_{2}$, and $x_{2}^{2}$ of both sides of Eq. (34), we have

$$
\left(\begin{array}{ccc}
-\frac{\theta}{2} & \eta & 0 \\
-\eta & -\theta & \eta \\
- & -\eta & -\frac{\theta}{2}
\end{array}\right)\left(\begin{array}{l}
a_{11} \\
a_{12} \\
a_{22}
\end{array}\right)=\left(\begin{array}{l}
\Omega_{1} \\
\Omega_{2} \\
\Omega_{3}
\end{array}\right)
$$


where

$$
\begin{aligned}
& \Omega_{1}=-\Delta\left(q_{32}\left(G_{4} p_{21}^{2}+G_{5} p_{21} p_{31}\right)+q_{33} G_{6} p_{21} p_{31}\right), \\
& \Omega_{2}=-\Delta\left(q_{32}\left(2 G_{4} p_{21} p_{22}+G_{5}\left(p_{21} p_{32}+p_{22} p_{31}\right)\right)+q_{33} G_{6}\left(p_{21} p_{32}+p_{22} p_{31}\right)\right), \\
& \Omega_{3}=-\Delta\left(q_{32}\left(G_{4} p_{22}^{2}+G_{5} p_{22} p_{32}\right)+q_{33} G_{6} p_{22} p_{32}\right) .
\end{aligned}
$$

It can be easily shown that

$$
\begin{aligned}
& a_{11}=-\frac{\left[\eta^{2}\left(\Omega_{1}+\Omega_{3}\right)+\frac{\theta}{2}\left(\eta \Omega_{2}+\theta \Omega_{1}\right)\right]}{\left(\frac{\theta^{3}}{4}+\eta^{2} \theta\right)}, \\
& a_{12}=-\frac{\left[\frac{\theta^{2} \Omega_{2}}{4}+\frac{\eta \theta}{2}\left(\Omega_{3}-\Omega_{1}\right)\right]}{\left(\frac{\theta^{3}}{4}+\eta^{2} \theta\right)}, \\
& a_{13}=-\frac{\left[\frac{\theta^{2} \Omega_{3}}{2}-\frac{\theta}{2} \eta \Omega_{2}+\eta^{2}\left(\Omega_{1}+\Omega_{3}\right)\right]}{\left(\frac{\theta^{3}}{4}+\eta^{2} \theta\right)} .
\end{aligned}
$$

Then the flow on center manifold is governed by the two-dimensional system

$$
\dot{x}=A x+F_{1}(x, \psi((x)) .
$$

Now we give the center manifold theorem by the following theorem to determine the asymptotic behaviors of the solution of (25).

Theorem 3.3 Suppose that the zero solution of (37) is asymptotically stable (unstable), then the zero solution of (25) is asymptotically stable (unstable).

System (37) can be written as

$$
\frac{d}{d t}\left(\begin{array}{l}
x_{1} \\
x_{2}
\end{array}\right)=\left(\begin{array}{cc}
0 & -\eta \\
\eta & 0
\end{array}\right)\left(\begin{array}{l}
x_{1} \\
x_{2}
\end{array}\right)+\left(\begin{array}{l}
\Gamma \\
\Sigma
\end{array}\right),
$$

where $\Gamma=q_{11} \varphi_{1}+q_{12} \varphi_{2}+q_{13} \varphi_{3}+o\left(\rho^{2}\right), \Sigma=q_{21} \varphi_{1}+q_{22} \varphi_{2}+q_{23} \varphi_{3}+o\left(\rho^{2}\right), \Lambda, \varphi_{1}, \varphi_{2}$, and $\varphi_{3}$ are given in (33).

The stability of the limit cycle arising from a Hopf bifurcation is determined by the sign of the quantity $\Pi$, where

$$
\begin{aligned}
\Pi= & \Gamma_{111}+\Sigma_{112}+\Gamma_{122}+\Sigma_{222}+\frac{1}{\eta}\left[\Gamma_{12}\left(\Gamma_{11}+\Gamma_{22}\right)\right. \\
& \left.-\Sigma_{12}\left(\Sigma_{11}+\Sigma_{22}\right)-\Gamma_{11} \Sigma_{11}+\Gamma_{22} \Sigma_{22}\right],
\end{aligned}
$$

where $\Gamma_{i j k}$ denotes the partial derivative $\frac{\partial^{3} \Gamma}{\partial x_{i} \partial x_{j} \partial x_{k}}$ at the origin and quantities with two subscripts represent order partial derivatives at the origin.

If $\Pi<0$, the bifurcation limit cycle is stable and the Hopf bifurcation is called supercritical; if $\Pi>0$, the bifurcation limit cycle is unstable and the Hopf bifurcation is called subcritical. 
Here

$$
\begin{aligned}
& \Gamma_{111}=-q_{12}\left(6 a_{11} G_{4} p_{21} p_{23}+3 a_{11} G_{5} p_{21} p_{23}+3 a_{11} G_{5} p_{23} p_{31}\right), \\
& \Sigma_{112}=-q_{22}\left(2 a_{11} G_{4} p_{22} p_{23}+a_{11} G_{5} p_{22} p_{23}+a_{11} G_{5} p_{23} p_{32}\right), \\
& \Gamma_{122}=-q_{12}\left(2 a_{22} G_{4} p_{21} p_{23}+a_{22} G_{5} p_{21} p_{33}+a_{22} G_{5} p_{23} p_{31}\right), \\
& \Sigma_{112}=-q_{22}\left(6 a_{11} G_{4} p_{22} p_{23}+3 a_{22} G_{5} p_{22} p_{33}+3 a_{22} G_{5} p_{23} p_{32}\right), \\
& \Gamma_{11}=-2 q_{12}\left(G_{4} p_{21}^{2}+G_{5} p_{21} p_{31}\right)-2 q_{13} G_{6} p_{21} p_{31}, \\
& \Gamma_{22}=-2 q_{12}\left(G_{4} p_{22}^{2}+G_{5} p_{22} p_{32}\right)-2 q_{13} G_{6} p_{22} p_{32}, \\
& \Sigma_{11}=-2 q_{22}\left(G_{4} p_{21}^{2}+G_{5} p_{21} p_{31}\right)-2 q_{23} G_{6} p_{21} p_{31}, \\
& \Sigma_{22}=-2 q_{22}\left(G_{4} p_{22}^{2}+G_{5} p_{22} p_{32}\right)-2 q_{23} G_{6} p_{22} p_{32}, \\
& \Gamma_{12}=-q_{12}\left[2 G_{4} p_{21}^{2}+G_{5}\left(p_{21} p_{32}+p_{22} p_{31}\right)\right]-q_{13} G_{6}\left(p_{21} p_{32}+p_{22} p_{31}\right), \\
& \Sigma_{12}=-q_{22}\left[2 G_{4} p_{21} p_{22}+G_{5}\left(p_{21} p_{32}+p_{22} p_{31}\right)\right]-q_{23} G_{6}\left(p_{21} p_{32}+p_{22} p_{31}\right) .
\end{aligned}
$$

The sign of $\Pi$ can be obtained by putting the values of $\Gamma_{111}, \Sigma_{112}, \Gamma_{122}, \Sigma_{222}, \Gamma_{11}, \Gamma_{22}, \Sigma_{11}$, $\Sigma_{22}, \Gamma_{12}, \Sigma_{12}$ in Eq. (39).

Based on the above results, for system (1), we have the following.

Theorem 3.4 If $R_{1}>1, R_{02}<1$, and $C_{1} C_{2}-C_{3}<0$, then for system (1), there exists a stable limit cycle in the $\left(M_{v}, S_{v}, P_{2}\right)$ space, in which case, despite oscillations, predation will lead to the elimination of the virus from the system, and uninfected vectors still exist.

Proof From the characteristic equation (9) of equilibrium $E_{3}$ of system (1), we have $\lim _{t \rightarrow \infty}\left(S_{h}(t), I_{h}(t), I_{v}(t)\right)=\left(S_{h}^{0}, 0,0\right)$ if $R_{1}>1$ and $R_{02}<1$. Then by Lemmas 3.2, 3.3 and Theorem 3.3 there exists a stable limit cycle in the $\left(M_{v}, S_{v}, P_{2}\right)$ space for system (1) if $R_{1}>1$, $R_{02}<1$ and $C_{1} C_{2}-C_{3}<0$.

This completes the proof of Theorem 3.4.

By numerical simulation, we show that there exists a stable limit cycle in the $\left(S_{h}, I_{h}, M_{v}\right.$, $\left.S_{v}, I_{v}, P_{2}\right)$ space for system (1) if $R_{1}>1, R_{02}>1$ and $C_{1} C_{2}-C_{3}<0$ (see Fig. 4-5).

\section{Numerical simulation of the system dynamics}

In this subsection, numerical simulations are given to show the stability and bifurcation of system (1) (see Fig. 1-5) and the effect of stage structure on disease dynamics with and without predators (see Fig. 6-7).

Example 1 We take $b_{1}=0.7978, b_{2}=0.9020, \mu_{h}=0.3485, \delta_{h}=0.8706, \mu_{v 1}=\mu_{v 2}=0.2381$, $d=0.8202, \beta_{1}=0.9050, \beta_{2}=0.6740, \alpha=0.1464$ (all the parameters are stochastically chosen for illustrative purpose only). Numerical calculations give $R_{01}=3.1489>1$ and $C_{1} C_{2}-C_{3}=-0.0138<0$. It follows from Corollary 3.1 that the disease equilibrium of system (2) is locally asymptotically stable, that is, the vector-borne disease will persist in the absence of predators (see Fig. 1).

Example 2 We take $a_{2}=0.6497, e_{2}=0.1356, \gamma_{2}=0.7855, h_{2}=0.6425$ and keep other parameters unchanged in Example 1. Numerical calculations give $R_{1}=6.2465>1$ and 

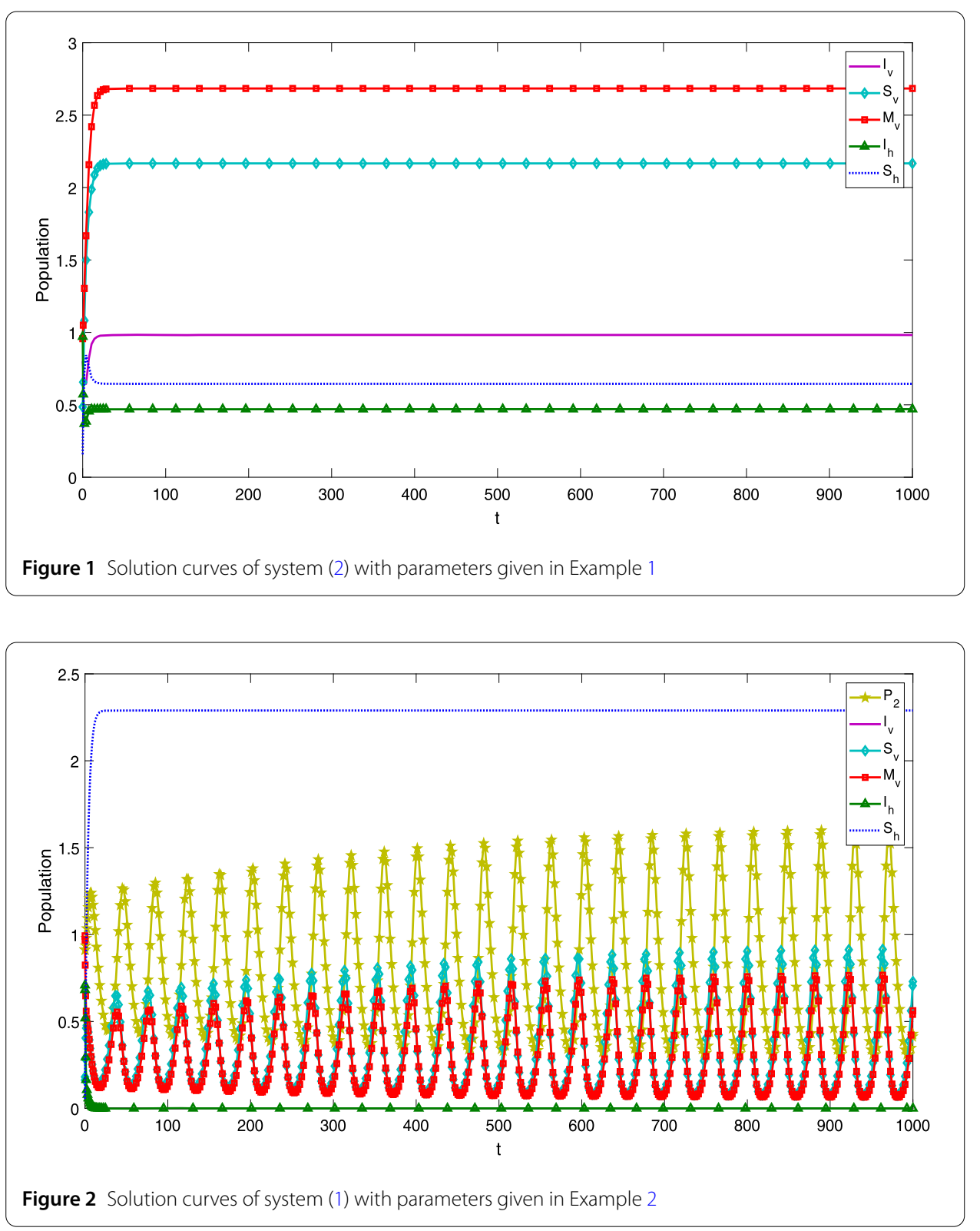

$R_{02}=0.5331<1$. It follows from Theorem 3.2 that the vector-borne disease will tend to die out (see Fig. 2). By calculations, $C_{1} C_{2}-C_{3}=-0.0138<0$, then by Theorem 3.4 there exists a stable limit cycle in the $\left(M_{v}, S_{v}, P_{2}\right)$ space for system (1) (see Fig. 3).

Example 3 Keep all the parameters fixed in Example 2 except for $h_{2}=0.3841$. By calculation the values of $R_{1}$ and $C_{1} C_{2}-C_{3}$ remain the same as in Example 2 and $R_{02}=1.0396>1$, then by Theorem 3.2 the vector-borne disease persists, but the equilibrium levels of the infected hosts and vectors have been greatly lessened when the predator $P_{2}$ is added (see Fig. 4). Moreover, Fig. 5 illustrates that there exists a stable limit cycle for system (1) if $R_{1}>1, R_{02}<1$, and $C_{1} C_{2}-C_{3}<0$.

Example 4 Take $\mu_{v 1}=0.1$ and keep all the parameters unchanged in Example 1 except for parameters $b_{2}$ and $d$. Then $R_{01}<1$ if $b_{2}$ and $d$ are close to 0.48 and 0.1, respectively. 


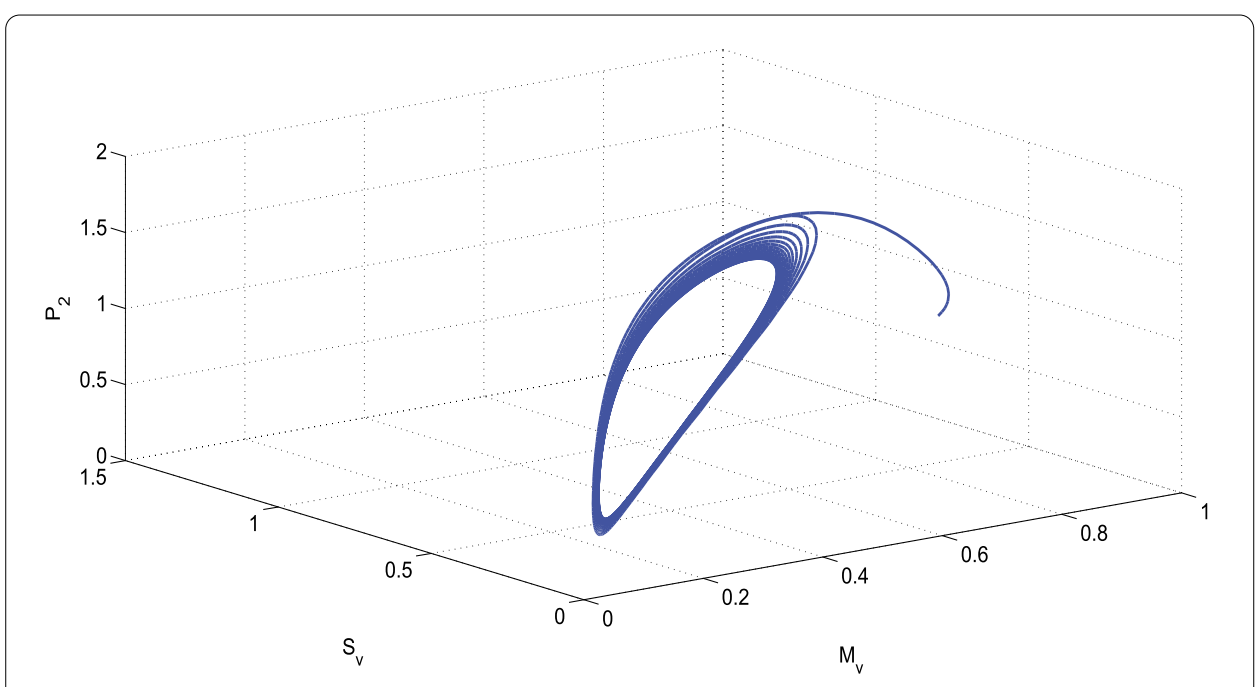

Figure 3 Stable limit cycle in the $\left(M_{v}, S_{v}, P_{2}\right)$ space for system (1) with parameters given in Example 2

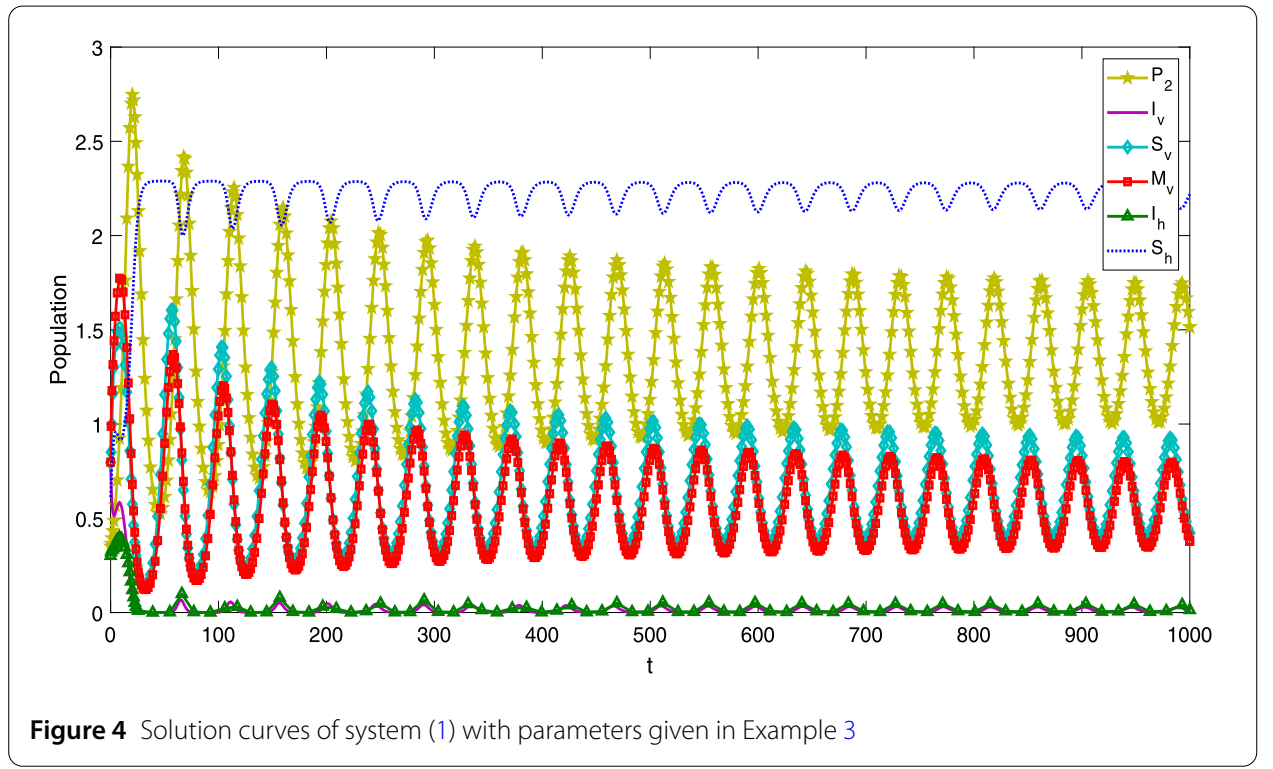

But if $b_{2}$ and $d$ are large, then we have $R_{01}>1$. This suggests that in the absence of the predator, the birth rate $b_{2}$ of the immature vectors and the conversion rate $d$ from the immature vectors to the mature vectors will cause the spread of the vector-borne disease (see the left-hand side of Fig. 6). Take $\mu_{\nu 1}=0.1$ and keep all the parameters unchanged in Example 2 except for parameters $b_{2}$ and $d$, then from the right-hand side of Fig. 6 it is clear that when predators are present, an increase in the vector birth rate $b_{2}$ and the conversion rate $d$ from the immature vectors to the mature vectors will reduce the value of the reproduction number $R_{02}$, and therefore lessen the prevalence of disease, because an increase in the vector birth rate and the conversion rate leads to an increase in the predator population.

Example 5 Take $b_{2}=0.7343$ and keep all the parameters unchanged in Example 4 except for parameters $\mu_{v 1}$ and $d$. Then $R_{01}<1$ if $d$ and $u_{v 1}$ are close to 0.1 and 0.2 , respectively. 

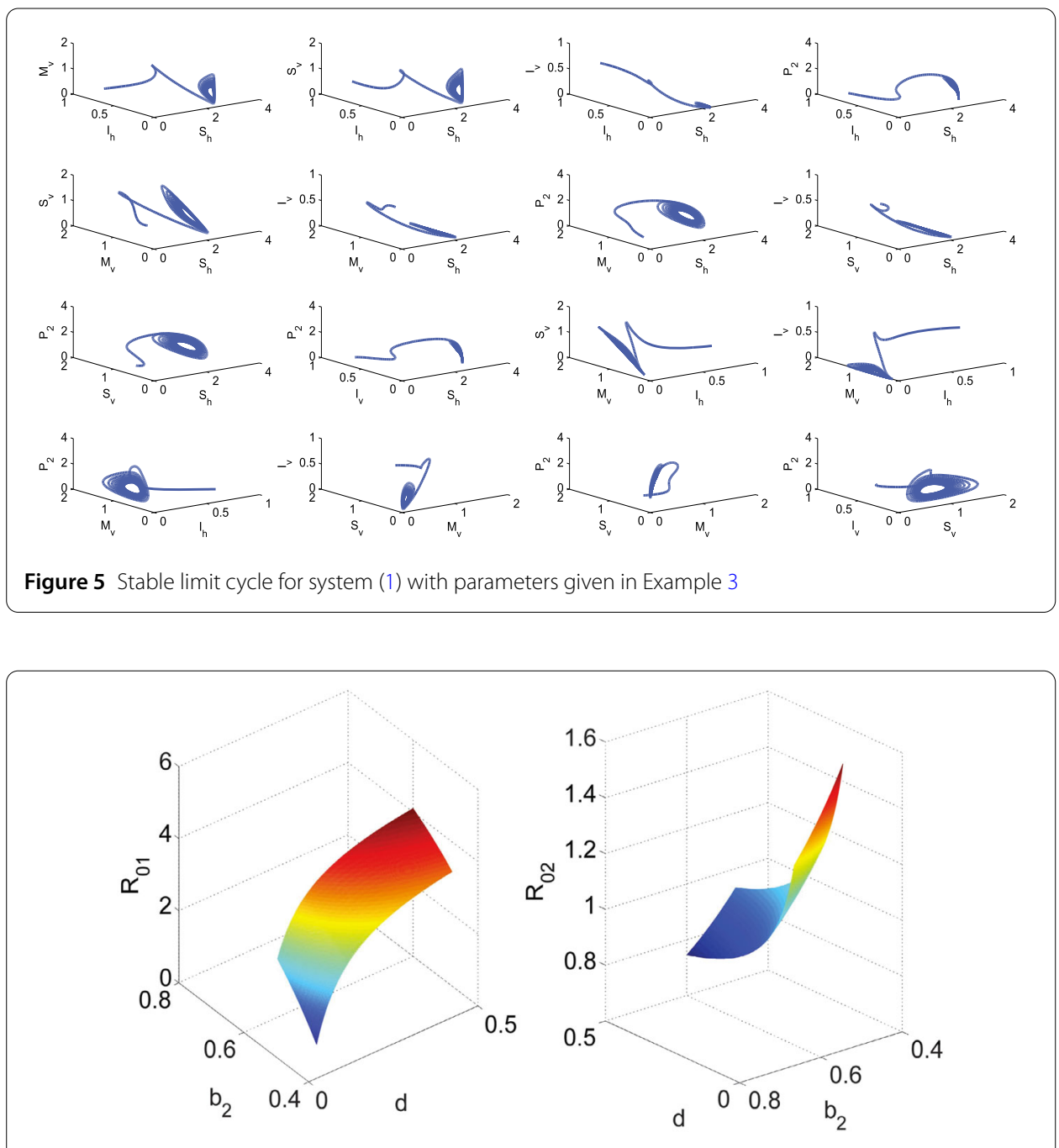

Figure 6 Surface plot of the basic reproduction numbers $R_{01}$ and $R_{02}$ as functions of $b_{2}$ and $d$ for the parameters given in Example 4

But if $d$ is large and $u_{v 1}$ is small, then we have $R_{01}>1$. This suggests that the conversion rate $d$ from the immature vectors to the mature vectors will cause the spread of the vectorborne disease in the absence of predators, while the natural death rate $u_{v 1}$ of the immature vector can reduce the vector disease spread (see the left-hand side of Fig. 7). When the predators are introduced, we find from the right-hand side of Fig. 7 that an increase in the conversion rate $d$ from the immature vectors to the mature vectors leads to a decline in the value of the reproduction number $R_{02}$, while an increase in the natural death rate of the immature vectors leads to an increase in the value of the reproduction number $R_{02}$, because an increase in the immature vector mortality rate $\mu_{v 1}$ indirectly leads to an increase in the predator densities.

Remark 2 From Figs. 2-3 and Figs. 4-5, it is clear that periodic solutions exist when a predator species $P_{2}$ is introduced in the system, suggesting the occurrence of a supercritical Hopf bifurcation. From Figs. 4-5, the hosts, the vectors, including the immature 


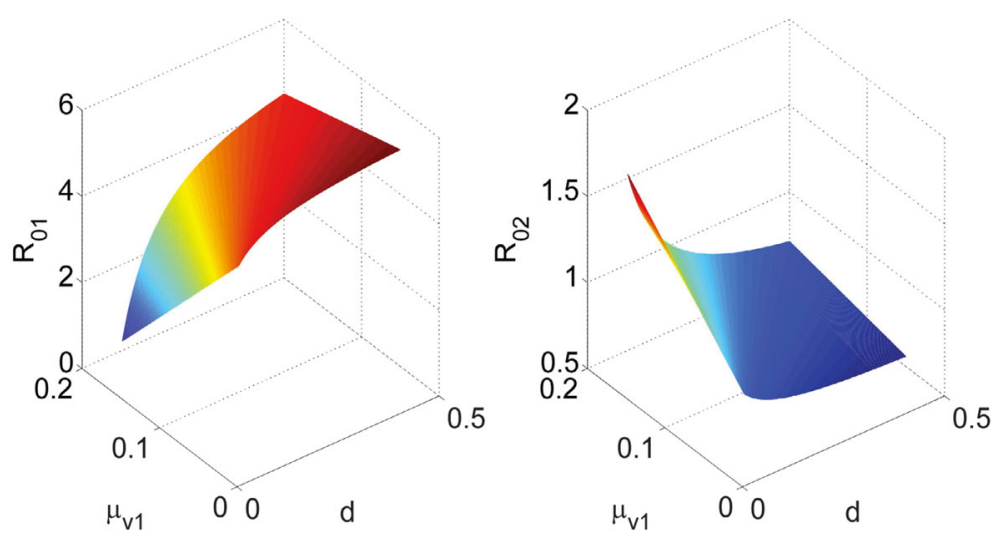

Figure 7 Surface plot of the basic reproduction numbers $R_{01}$ and $R_{02}$ as functions of $\mu_{v 1}$ and $d$ for the parameters given in Example 5

and mature ones, and the predators oscillate, leading to the persistence of the vectortransmitted disease when the predator species $P_{2}$ is added. By enhancing predators' attack rate $h_{2}$, the level of infected hosts $I_{h}$ and vectors $I_{v}$ will decline, and when $h_{2}=0.6425$, from Figs. 2-3 we can see that predation brings about the extinction of the disease, despite the oscillations between the predators and the vectors. That is to say, introducing predators to vectors is advantageous to vector-borne disease control, and the larger the capturing rate $h_{2}$ is, the better control of disease will be.

Remark 3 From the left-hand sides of Figs. 6 and 7, we find that the stage structure has different effects on disease transmission dynamics with and without predators. When predators are introduced, small values of the birth rate $b_{2}$ and the conversion rate $d$, and large value of the natural death rate $\mu_{v 1}$ will lead to the increase in the value of the basic reproduction number $R_{02}$, and therefore increase the risk of disease spread. In the absence of predators, we find that the reproduction number $R_{01}$ increases with the increase in the birth rate $b_{2}$ and the conversion rate $d$ of the immature vectors, and with the decrease in the natural death rate $\mu_{v 1}$ of the immature vectors. Therefore, for vector-host disease prevention and control in the absence of the predator, it is necessary to take some strategies to reduce the birth rate $b_{2}$ and the conversion rate $d$, and enlarge the natural death rate $\mu_{\nu 1}$ at vectors' larva stage, such as use of physical strategies, use of pesticides, biological control, and so on.

From Sect. 3 , we find that the predator $P_{2}$ brings about complicated dynamics of system (1), and we also find that introducing the predator has certain benefits to disease control. It is clear from Figs. $2-5$ that the larger the value of capturing rate $h_{2}$ is, the lower equilibrium levels of infected hosts and vectors will be.

\section{Conclusions and discussions}

In this paper, we mainly formulate and analyze a host-vector-predator model with stage structure for the vectors. The period of growth for the vectors is divided into immature and mature stages according to the transmission properties (e.g., cysticercosis and scrub typhus are spread among hosts by the immature vectors, while some infectious diseases, such as malaria, dengue fever, West Nile virus, and pine wilt disease, are transmitted 
among hosts by the mature vectors). The interactions between the predator and the vector are modeled by Holling type II functional response. Corollary 3.1 reveals that in the absence of the predator, the reproduction number $R_{01}$ of system (2) provides the threshold which decides the persistence and extinction of vector-borne diseases. If $R_{01}<1$, the diseases will tend to die out, while if $R_{01}>1$ then the diseases will persist (see Fig. 1 ). When the predators are added, we find from Theorem 3.2 that the diseases still persist if $R_{02}>1$, but the equilibrium levels of the infected hosts and vectors have been lessened (see Fig. 2). If $R_{02}<1$, then by Theorem 3.2 the diseases can be eradicated (see Fig. 3 ). Therefore, predators have positive effects on disease control. Moreover, by Theorem 3.2 predation causes the phenomenon of limit cycle arising from a Hopf bifurcation (see Fig. 2-5). If $R_{02}<1$ and $C_{1} C_{2}-C_{3}>0$, then there exists a stable limit cycle in the $\left(M_{v}, S_{v}, P\right)$ space for system (1). Though periodic oscillation occurs between the vectors and the predators, the diseases can be eradicated by the effect of predators on vectors (see Fig. 2-3). However, if $R_{02}>1$ and $C_{1} C_{2}-C_{3}>0$, then there exists a stable limit cycle for system (1), and the diseases cannot be eradicated (see Fig. 4-5). Finally, the effect of stage structure on disease spread with and without predators has been illustrated (see Fig. 6-7). From the left-hand sides of Figs. 6 and 7, it is clear that in the absence of predators, the reproduction number $R_{01}$ increases with the increase in the birth rate $b_{2}$ and the conversion rate $d$ of the immature vectors, while $R_{01}$ increases with the decrease in the natural death rate $\mu_{v 1}$ of the immature vectors. Therefore, it is necessary to take some strategies to reduce the birth rate $b_{2}$ and the conversion rate $d$ and enlarge the natural death rate $\mu_{v 1}$ at vectors' larva stage through the use of physical strategies, use of pesticides, biological control, and so on. From the right-hand sides of Figs. 6 and 7, we find that after introducing the predators, the effect of $b_{2}, d$, and $\mu_{v 1}$ on disease transmission is inverse compared with the effect of $b_{2}, d$, and $\mu_{v 1}$ on disease transmission in the absence of predators. Therefore, to exert the best effect of predators on disease control, we should increase the values of the birth rate $b_{2}$ and the conversion rate $d$ to certain degree, and decrease the value of the natural death rate $\mu_{v 1}$ of vectors at the immature stage.

\section{Acknowledgements}

The authors would like to thank the editor and two anonymous reviewers for their valuable suggestions and comments.

Funding

The work is supported by the National Natural Science Foundation of China (grant no. 71701082 and 71271103), the Six Talents Peak Foundation of Jiangsu Province, and the Innovative Foundation for Doctoral Candidate of Jiangsu Province, China (grant no. CXZZ13_0687).

Competing interests

The authors declare that they have no competing interests.

Authors' contributions

All authors contributed equally to the writing of this paper. All authors read and approved the final manuscript.

Author details

${ }^{1}$ Department of Mathematics, Shaoxing University, Shaoxing, P.R. China. ${ }^{2}$ School of Mathematics and Statistics, Central South University, Changsha, P.R. China. ${ }^{3}$ Faculty of Science, Jiangsu University, Zhenjiang, P.R. China. ${ }^{4}$ College of Civil Engineering, Shaoxing University, Shaoxing, P.R. China. ${ }^{5}$ School of Finance and Economics, Jiangsu University, Zhenjiang, P.R. China.

\section{Publisher's Note}

Springer Nature remains neutral with regard to jurisdictional claims in published maps and institutional affiliations. 


\section{References}

1. Jones, K.E., Patel, N.G., Levy, M.A., Storeygard, A., Balk, D., Gittleman, J.L., Daszak, P.: Global trends in emerging infectious diseases. Nature 451, 990-993 (2008)

2. Daszak, P., Cunningham, A., Hyatt, A.D.: Anthropogenic environmental change and the infectious diseases in wildlife. Acta Trop. 78, 103-116 (2001)

3. Yang, C.X., Nie, L.F.: Modelling the use of impulsive vaccination to control Rift Valley Fever virus transmission. J. Differ. Equ. 2016, 134 (2016)

4. Nie, L.F., Xue, Y.N.: The roles of maturation delay and vaccination on the spread of Dengue virus and optimal control. J. Differ. Equ. 2017, 278 (2017)

5. Hemingway, J., Ranson, H.: Insecticide resistance in insect vectors of human disease. Annu. Rev. Entomol. 45, 371-391 (2000)

6. Castro, M.C., De Yamagata, Y., Mtasiwa, D., Tanner, M., Utzinger, J., Keiser, J., Singer, B.H.: Integrated urban malaria control: a case study in Dares Salaam, Tanzania. Am. J. Trop. Med. Hyg. 71, 103-117 (2004)

7. Benedict, M.Q., Robinson, A.S.: The first releases of transgenic mosquitoes: an argument for the sterile insect technique. Trends Parasitol. 19, 349-355 (2003)

8. Kaaya, G.P., Hassan, S.: Entomogenous fungi as promising biopesticides for tick control. Exp. Appl. Acarol. 24,913-926 (2000)

9. Scholte, E.J., Ng'Habi, K., Kihonda, J., Takken, W., Paaijmans, K., Abdulla, S., Killeen, G.F., Knols, B.G.J.: An entomopathogenic fungus for control of adult African malaria mosquitoes. Science 308, 1641-1642 (2005)

10. Legner, E.: Biological control of Diptera of medical and veterinary importance. J. Vector Ecol. 20, 59-120 (1995)

11. Stauffer, J.R. Jr., Arnegard, M., Cetron, M.: Controlling vectors and hosts of parasitic diseases using fishes. Bioscience 47, 41-49 (1997)

12. Samish, M., Rehacek, J.: Pathogens and predators of ticks and their potential in biological control. Annu. Rev. Entomol. 44, 159-182 (1999)

13. Ostfeld, R.S., Price, A., Hornbostel, V.L., Benjamin, M.A., Keesing, F.: Controlling ticks and tick-borne zoonoses with biological and chemical agents. Bioscience 56, 383-394 (2006)

14. Walker, K., Lynch, M.: Contributions of Anopheles larval control to malaria suppression in tropical Africa: review of achievements and potential. Med. Vet. Entomol. 21, 2-21 (2007)

15. Ghosh, S.K., Tiwari, S.N., Sathyanarayan, T.S., Sampath, T.R., Sharma, V.P., Nanda, N., Joshi, H., Adak, T., Subbarao, S.K.: Larvivorous fish in wells target the malaria vector sibling species of the Anopheles culicifacies complex in villages in Karnataka, India. Trans. R. Soc. Trop. Med. Hyg. 99, 101-105 (2005)

16. Ghosh, S.K., Dash, A.P.: Larvivorous fish against malaria vectors: a new outlook. Trans. R. Soc. Trop. Med. Hyg. 101, 1063-1064 (2007)

17. Kay, B., Nam, V.S.: New strategy against Aedes aegypti in Vietnam. Lancet 365, 613-617 (2005)

18. Kittayapong, P., Yoksan, S., Chansang, U., Chansang, C., Bhumiratana, A.: Suppression of dengue transmission by application of integrated vector control strategies at sero-positive GIS-based foci. Am. J. Trop. Med. Hyg. 78, 70-76 (2008)

19. Zhang, L.Q., Liu, J., Wu, H.: The screening of a virulent strain of Beauveria bassiana to Monochamus alternatus. J. Nanjing For. Univ. 24, 33-37 (2000)

20. Lai, Y.X., Liu, J.D., Xu, Q.Y., Wang, Y.H., Zhou, C.M.: Trials on the parasitism of Beauveria bassiana or Verticillium lecanii on larvae of Monochamus alternatus Hope. J. Jiangsu. For. Sci. Technol. 30, 7-9 (2003)

21. Jeger, M.J., Holt, J., Van Den Bosch, F., Madden, L.V.: Epidemiology of insect-transmitted plant viruses: modelling disease dynamics and control interventions. Physiol. Entomol. 29, 291-304 (2004)

22. Otim, M., Legg, D., Kyamanywa, S., Polaszek, A., Gerling, D.: Population dynamics of Bemisia tabaci (Homoptera: Aleyrodidae) parasitoids on cassava mosaic disease-resistant and susceptible varieties. Biocontrol Sci. Technol. 16 205-214 (2006)

23. Luck, R.F., Shepard, B.M., Kenmore, P.E.: Experimental methods for evaluating arthropod natural enemies. Annu. Rev. Entomol. 33, 367-389 (1988)

24. Zehnder, G., Gurr, G.M., Kühne, S., Wade, M.R., Wratten, S.D., Wyss, E.: Arthropod pest management in organic crops. Annu. Rev. Entomol. 52, 57-80 (2007)

25. Tian, B.D., Yang, L., Zhong, S.M.: Global stability of a predator-prey model with Allee effect. Int. J. Biomath. 8, 37-51 (2015)

26. Zha, L.J., Cui, J.A., Zhou, X.Y.: Ratio-dependent predator-prey model with stage structure and time delay. Int J. Biomath. 5, 15-37 (2012)

27. Zhou, F.Y.: Existence and global attractivity of a positive periodic solution for a non-autonomous predator-prey mode under viral infection. Int. J. Biomath. 2, 419-442 (2009)

28. Schraiber, J.G., Kaczmarczyk, A.N., Kwok, R., et al.: Constraints on the use of lifespan-shortening Wolbachia to control dengue fever. J. Theor. Biol. 297, 26-32 (2012)

29. Hughes, H., Britton, N.F.: Modelling the use of Wolbachia to control dengue fever transmission. Bull. Math. Biol. 75, 796-818 (2013)

30. Moore, S.M., Borer, E.T., Hosseini, P.R.: Predators indirectly control vector-borne disease: linking predator-prey and host-pathogen models. J. R. Soc. Interface 7, 161-176 (2009)

31. Zhou, F.Y., Yao, H.Y.: Dynamics and biocontrol: the indirect effects of a predator population on a host-vector disease model. Abstr. Appl. Anal. 2014, 252718 (2014)

32. Okamoto, K.W., Amarasekare, P.: The biological control of disease vectors. J. Theor. Biol. 309, 47-57 (2012)

33. Xiao, Y.N., Chen, L.S.: An SIS epidemic model with stage structure and a delay. Acta Math. Appl. Sin. 18, 607-618 (2002)

34. Cai, L., Li, X., Ghosh, M.: Global stability of a stage-structured epidemic model with a nonlinear incidence. Appl. Math. Comput. 214, 73-82 (2009)

35. Hyman, J.M., Li, J., Stanley, E.A.: The differential infectivity and staged progression models for the transmission of HIV. Math. Biosci. 155, 77-109 (1999)

36. Moghadas, S.M., Gumel, A.B.: Global stability of a two-stage epidemic model with generalized non-linear incidence. Math. Comput. Simul. 60, 107-118 (2002) 
37. Martcheva, M., Castillo-Chavez, C.: Diseases with chronic stage in a population with varying size. Math. Biosci. 182, $1-25(2003)$

38. Tian, B.D., Jin, Y.G., Zhong, S.M., Chen, N.: Global stability of an epidemic model with stage structure and nonlinear incidence rates in a heterogeneous host population. J. Differ. Equ. 2015, 260 (2015)

39. World health organization: Frequently Asked Questions Scrub Typhus (2008)

40. Zhao, B.G., Futai, K., Jack, R., Sutherland, J.R., Takeuchi, Y.: Pine Wilt Disease. Springer, New York (2008)

41. Diekmann, O., Heesterbeek, J.A.P., Metz, J.A.J.: On the definition and the computation of the basic reproduction ratio $R_{0}$ in models for infectious diseases in heterogeneous populations. J. Math. Biol. 28, 365-382 (1990)

42. Driessche, V.P., Watmough, J.: Reproduction numbers and sub-threshold endemic equilibria for compartmental models of disease transmission. Math. Biosci. 180, 29-48 (2002)

43. Singh, B.K., Chattopadhyay, J., Sinha, S.: The role of virus infection in a simple phytoplankton zooplankton system. J. Theor. Biol. 231, 153-166 (2004)

\section{Submit your manuscript to a SpringerOpen ${ }^{\circ}$ journal and benefit from:}

- Convenient online submission

- Rigorous peer review

- Open access: articles freely available online

- High visibility within the field

- Retaining the copyright to your article

Submit your next manuscript at $\gg$ springeropen.com 